\title{
Simulation of OLEDs with a polar Electron Transport Layer
}

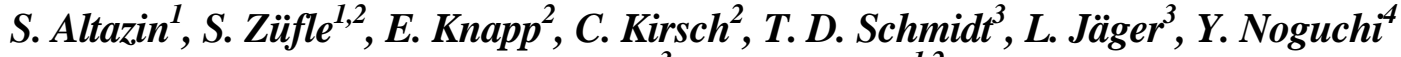 \\ W. Brïtting ${ }^{3}$, B. Ruhstaller ${ }^{1,2}$ \\ ${ }^{1}$ Fluxim AG, Winterthur, Switzerland \\ ${ }^{2}$ Zurich University of Applied Sciences, Institute of Computational Physics, Winterthur, Switzerland \\ ${ }^{3}$ Experimental Physics IV, Institute of Physics, University of Augsburg, Germany \\ ${ }^{4}$ School of Science and Technology, Meiji University, Kawasaki 214-8571, Japan
}

Organic light-emitting diodes (OLEDs) rely on the use of functional materials with suitable energy levels and mobilities for selective charge carrier injection and transport of one species only at the respective electrode. Until recently, however, the dipolar nature of many organic semiconductors has been largely ignored in this context. In particular, electron transports layers (ETLs) often exhibit spontaneous orientation polarization leading to interfacial charges that modify the electrical potential landscape inside a hetero-layer device.

Here we demonstrate that the effect of polar ETLs can be simulated using the well-established Poisson and drift-diffusion formalism, if these interfacial charges are taken into account. Impedance spectroscopy is used in order to validate our approach and to characterize the polarity of the material. Finally, simulations allow to quantify the impact of polar ETLs on device performance.

\section{Introduction}

OLED technology is gaining ground as next generation device for display and lighting applications. For mobile devices in display and lighting, the power efficiency is one of the most important parameters. Several paths are followed in order to increase the efficiency of OLEDs: both optical (like light scattering for lighting applications [1]) and electrical, for example using highly conductive hole transport layers (HTLs) and electron transport layers (ETLs) in order to decrease the operation voltage of the device. ETL materials often show a non-zero permanent molecular dipole moment. This behavior has been found in the two most common ETL materials, Tris(8-hydroxyquinolinato) aluminium ( $\mathrm{Alq}_{3}$ ) [2] and 4,7-diphenyl-1,10phenanthroline (BPhen) [3], and also in many others, as listed in [4]. When deposited in a thin film stack configuration, the polar ETL molecules do not arrange fully randomly, they have a slightly preferable orientation which effectively leads to a layer with a positive sheet charge density on one side and a negative one on the other side (see figure 1). The origin of this non-random orientation is however still not clear. So far, only experimental studies have been performed to investigate the impact of the polarity on the device operation, and only compact models based on equivalent circuits have been proposed to simulate such devices $[2,5]$. For the first time we show that the well-established drift-diffusion formalism implemented in SETFOS 4.3 [6] is able to cope with these materials leading to quantitative simulations of OLEDs embedding a polar ETL material. This allows us to both simulate the specific behavior introduced by such polar materials but also to give directions to improve the device operation. In this contribution we will mainly study a traditional bilayer OLED as shown in figure 1, but similar effects and conclusions can be found for more complex dye-doped multilayer devices [3]. In this contribution we make an extensive use of impedance spectroscopy characterization and modelling. Indeed this technique is known to be highly sensitive to the charges in the device; therefore this characterization technique is well suited to study the effect of polar 
materials on the device operation.

In section 2 we will introduce the basics of impedance spectroscopy as well as the basics of the mathematical model. In the second part, we will compare the simulation results with experimental characterization of OLEDs embedding a polar ETL material. This allows us to benchmark our approach and to extract some device and materials parameters. Finally, in a last part we will use our model to simulate the potential benefit of the ETL polarity on the IV curve of the device.
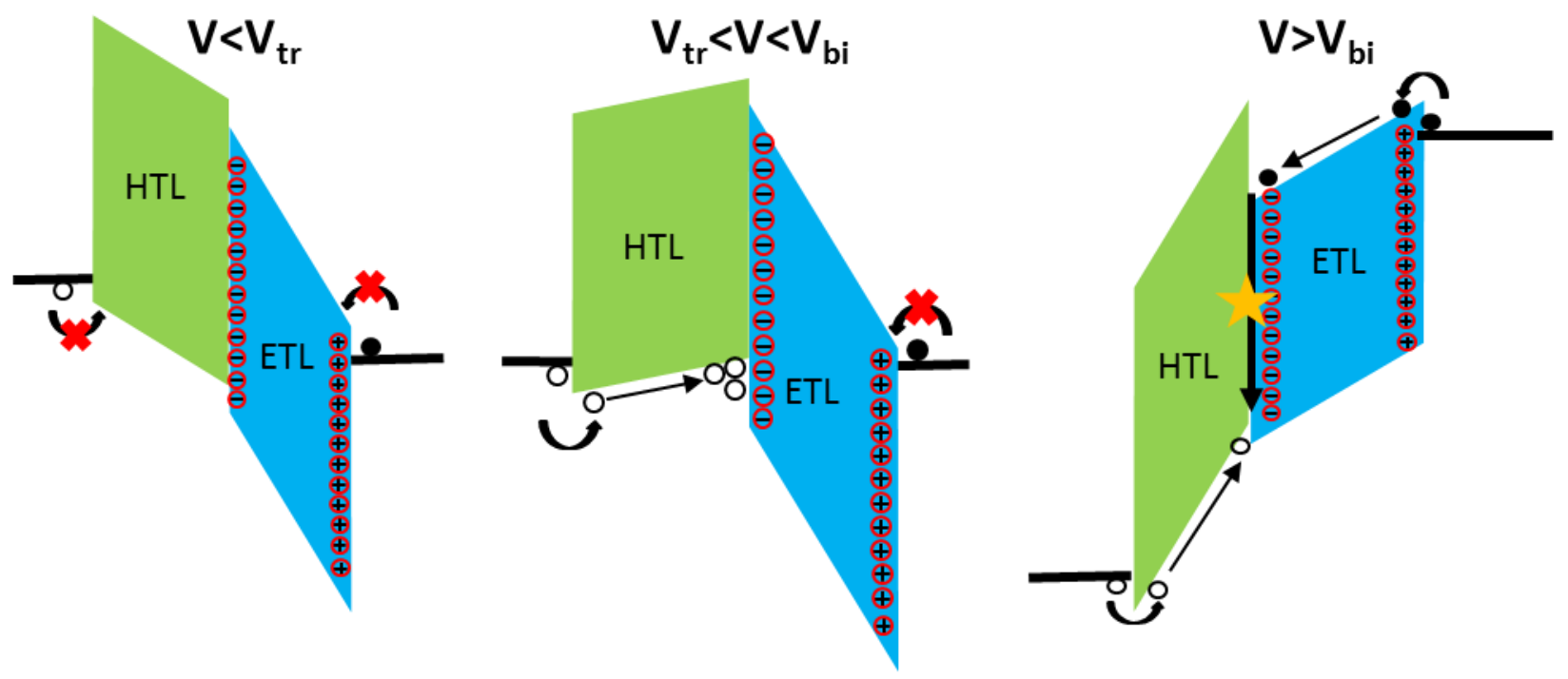

FIG. 1. Schematic band diagram of the bilayer OLED used in this study. The ETL $\left(\mathrm{Alq}_{3}\right)$ layer is supposed to have a molecular polar moment that is not randomly oriented, resulting in sheet charge densities on either side of the layer. This sheet charge density modifies the band diagram.

\section{Modelling of polar materials by drift-diffusion simulations}

Impedance spectroscopy is a well-known technique to study OLED device operation. It has been extensively used to characterize the doping concentration [7] or the mobility [8, 9] in organic and inorganic materials, as well as self-heating $[10]$.

In this technique, the device is biased at a constant voltage $\mathrm{V}_{0}$, on top of which small sinusoidal voltage $\left(\mathrm{V}_{1}\right)$ is applied. The total voltage $(\mathrm{V})$ applied to the device is then:

$V=V_{0}+V_{a c} \cdot \sin (2 \pi f t)$

Here $\mathrm{f}$ represents the frequency and the time. The basic concept of impedance spectroscopy is that the voltage $\mathrm{V}_{\mathrm{ac}}$ is small enough such that the device behaves in a linear regime. Therefore the admittance of the device can be written as: 
$Y=J_{a c} / V_{a c}=G+i \omega C$

Where $\mathrm{J}_{\mathrm{ac}}$ represents the current flowing out of the device minus the steady state current and $\mathrm{V}_{\mathrm{ac}}=V_{1}$. $\exp (i 2 \pi f t)$

Finally, an equivalent capacitance $\mathrm{C}$ and conductance $\mathrm{G}$ of the device can be calculated. By varying the frequency $\mathrm{f}$, the capacitance versus frequency of the device can be measured, and by varying the constant voltage $\mathrm{V}_{0}$ one can measure the well-known C-V response of the device.

In order to simulate the device operation we use the well-established physical drift-diffusion formalism implemented in SETFOS 4.3. It consists of the continuity equation for electrons and holes coupled with the Poisson equation:

$\operatorname{div}\left(-\mu_{n} \cdot \boldsymbol{n} \cdot \vec{E}-\frac{k_{b} T}{q} \mu_{n} \cdot \overrightarrow{\operatorname{grad}}(\boldsymbol{n})\right)-R=-\frac{\partial \boldsymbol{n}}{\partial t}$

$\operatorname{div}\left(\mu_{p} \cdot \boldsymbol{p} \cdot \vec{E}-\frac{k_{b} T}{q} \mu_{p} \cdot \overrightarrow{\operatorname{grad}}(\boldsymbol{p})\right)-R=-\frac{\partial \boldsymbol{p}}{\partial t}$

$\Delta \mathbf{V}=-\frac{q}{\varepsilon}(\boldsymbol{p}-\boldsymbol{n}+Q)$,

where $\mu_{\mathrm{n}, \mathrm{p}}$ represents the mobility of electrons (n) and holes (p), respectively, $\mathrm{V}$ the electrostatic potential, $\mathrm{R}$ the Langevin recombination rate and $\mathrm{Q}$ the electrostatic fixed charge. This latter quantity is of primary importance for the simulation of polar materials: as explained previously, the non-zero permanent dipole moment of the molecules associated with a preferred orientation during the deposition process effectively leads to a layer with a positive sheet charge density on one side and a negative one on the other side. In our simulations, the polar ETL is thus modelled by introducing two thin layers with an opposite charge density on the sides of the ETL. For the sake of simplicity, we assume in the simulations that the ETL molecules do not rearrange for the different applied voltages, which is supported by the stable capacitance at low frequencies. A rearrangement of the permanent dipole moments leading to an effective decrease of the sheet charge density has however been found to take place during degradation [3]. In order to take into account the internal barriers between the two organic materials, the continuity of the quasi fermi levels of both holes and electrons is postulated in the simulations [6,11], moreover the alignment of the Fermi levels at the metal/organic interfaces is assumed as a boundary condition.

Finally, in order to simulate impedance spectroscopy, the three variables: $\mathbf{n}, \mathbf{p}$ and $\mathbf{V}$ are replaced by [12]:

$$
\begin{aligned}
& n=n_{e q}+\boldsymbol{n}_{\boldsymbol{a c} \cdot} \cdot e^{i 2 \pi f t} \\
& p=p_{e q}+\boldsymbol{p}_{\boldsymbol{a c} \cdot} \cdot e^{i 2 \pi f t} \\
& V=V_{e q}+\boldsymbol{V}_{\boldsymbol{a c} \cdot} \cdot e^{i 2 \pi f t}
\end{aligned}
$$




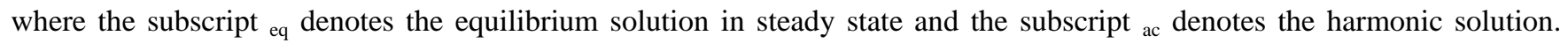
Then, assuming that the variables $\mathrm{n}_{\mathrm{ac}}, \mathrm{p}_{\mathrm{ac}}$ and $\mathrm{V}_{\mathrm{ac}}$ are small enough (which is the basic assumption of impedance spectroscopy), the system is linearized and solved. The quantity $\mathrm{J}_{\mathrm{ac}}$, which represents the harmonic current flowing out of the device, can then be calculated as well as the equivalent admittance of the device. The approach described above has been implemented in the software SETFOS 4.3 [6] and allows for fast and reliable impedance spectroscopy simulations without having to solve the equations in a time domain regime which would be much more time consuming.

\section{Comparison between simulations and experiments}

\subsection{Capacitance-Voltage characteristics}

The Capacitance-Voltage experiment $(\mathrm{C}-\mathrm{V})$ is known to be sensitive to the charge distribution in the device. Previous studies of Brütting et al. [2] have shown that the polar nature of $\mathrm{Alq}_{3}$ induces a typical signature in the $\mathrm{C}-\mathrm{V}$ experiment. Indeed, as in [2], and in figure 2 a)) two plateaus can be observed in the C-V plot. Interestingly, the transition voltage ( $\left.\mathrm{V}_{\text {tr }}\right)$, at which the capacitance changes, is directly related to the thickness of the $\mathrm{Alq}_{3}$ layer. This transition voltage occurs at negative values of the applied bias, suggesting the presence of mobile charges in the device at voltages below the $\mathrm{V}_{\mathrm{bi}}($ built in voltage $\approx 2 \mathrm{~V})$.

We find that $\mathrm{C}-\mathrm{V}$ simulations of such devices, using the approach developed in the previous section, can nicely reproduce this experimental observation (figure $2 \mathrm{~b}$ )). In order to simulate the polar nature of the $\mathrm{Alq}_{3}$, a negative sheet charge density of $\sigma=1.2 \mathrm{mC} / \mathrm{m}^{2}$ was introduced at the interface between $\mathrm{Alq}_{3}$ and $N, N^{\prime}$-Bis(3-methylphenyl)- $N, N^{\prime}$-diphenylbenzidine (TPD) and a positive one at the interface with the Calcium electrode This value is in nice agreement with the one found in [13] using a Kelvin probe measurement.
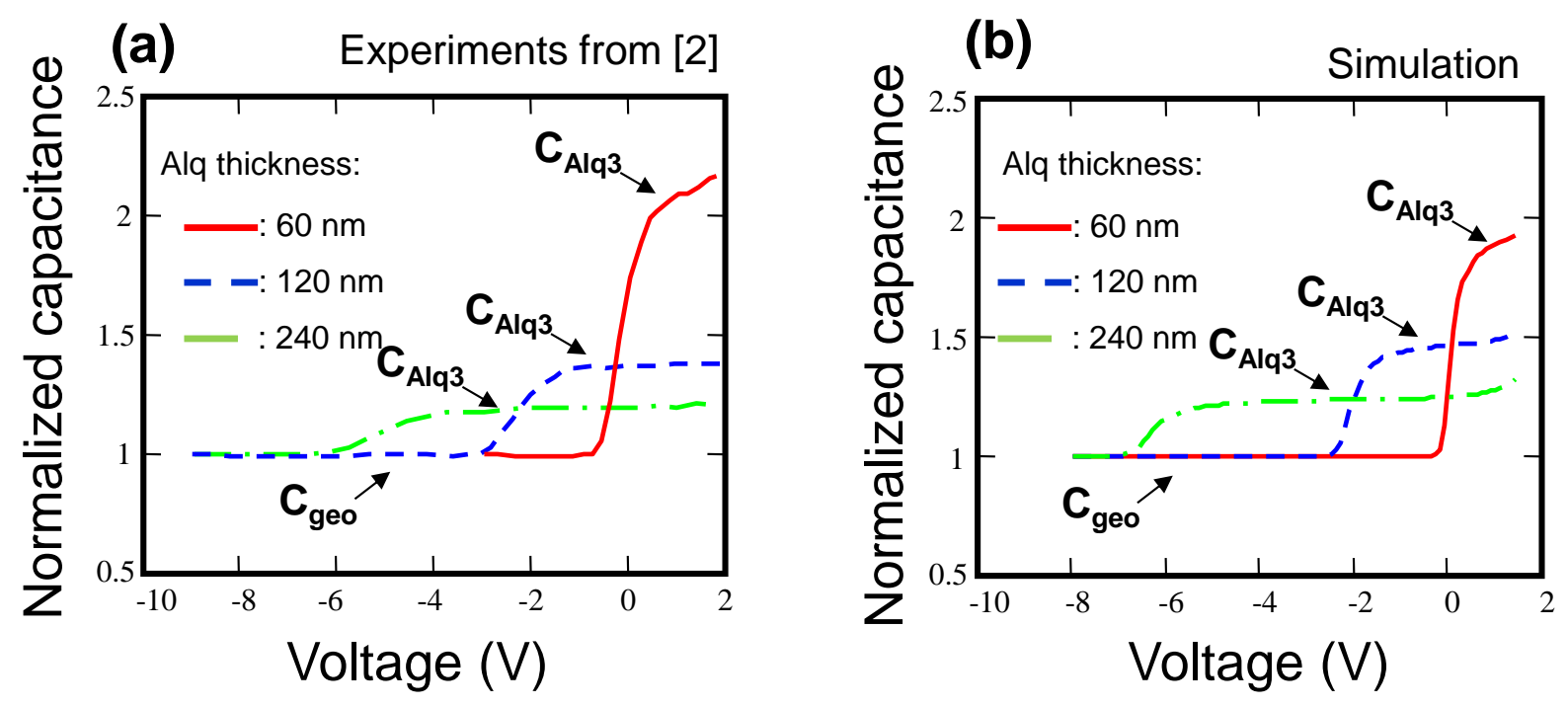


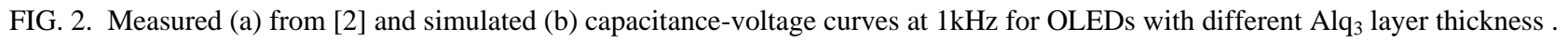

In order to be sure that the steps in the $\mathrm{C}-\mathrm{V}$ measurements and simulations (capacitance increase below the turn-on voltage) are a consequence of the polarity of the material, we have run the same simulations assuming, this time, no fixed charges in the device $(\mathrm{Q}=0)$ (see supplementary information). As expected, the simulated $\mathrm{C}-\mathrm{V}$ curves did not show any features below $\mathrm{V}_{\mathrm{bi}}$.

In order to understand and explain the different regimes observed in the $\mathrm{C}-\mathrm{V}$ curve we have simulated the hole and electron concentration in the device in three different regimes (Figure 3) namely for $\mathrm{V}\left\langle\mathrm{V}_{\mathrm{tr}}\right.$ (red), for $\mathrm{V}_{\mathrm{tr}}<\mathrm{V}<\mathrm{V}_{\mathrm{bi}}$ (blue) and for $\left.\mathrm{V}\right\rangle \mathrm{V}_{\mathrm{bi}}$ (green) for a device with a thick ETL (240nm) and with a HTL of $60 \mathrm{~nm}$.
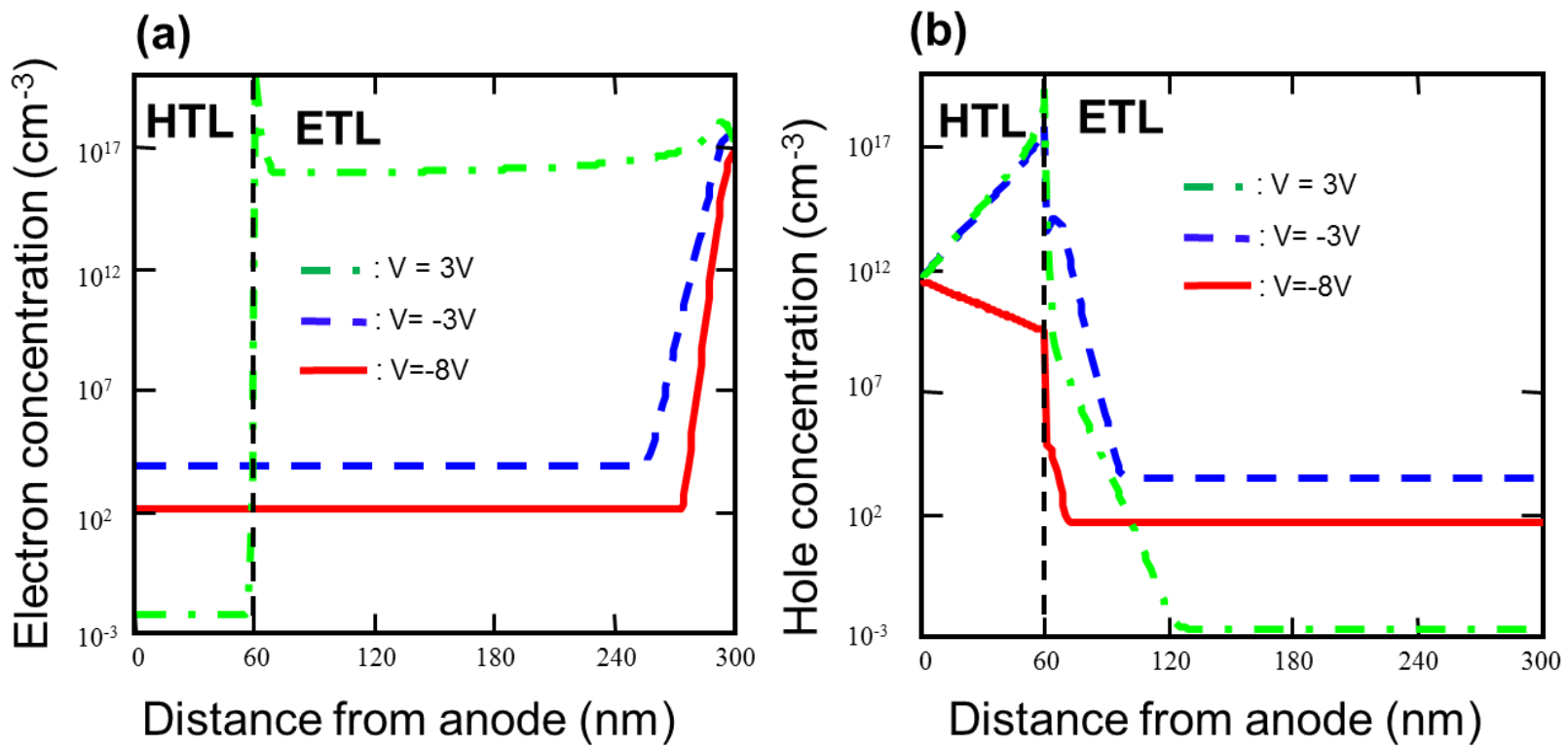

Fig. 3: Simulated electron (a) and hole (b) concentration profiles in the device for the three operation regimes described in the text.

One can see that for $\mathrm{V}=-8 \mathrm{~V}\left(\mathrm{~V}<\mathrm{V}_{\mathrm{tr}}\right)$ no holes or electrons are present in the device, the OLED behaves as a perfect insulator, the capacitance that is simulated and characterized is therefore equal to the geometrical capacitance of the full OLED: $C_{g e o}=\frac{\varepsilon_{E T L} \cdot \varepsilon_{H T L}}{\varepsilon_{E T L} \cdot t_{H T L}+\varepsilon_{H T L} \cdot t_{E T L}}$

Where $\varepsilon_{E T L / H T L}$ represents the dielectric constant of the ETL/HTL and $t_{E T L / H T L}$ represents the thickness of the ETL/HTL.

For $\mathrm{V}=-3 \mathrm{~V}$, and thus $\mathrm{V}_{\mathrm{tr}}<\mathrm{V}<\mathrm{V}_{\mathrm{bi}}$, holes are injected in the TPD layer. Indeed due to the presence of negative charges at the interface between the HTL and the ETL, the electric potential and therefore the band diagram is impacted leading to an injection of holes into the HTL for negative bias (see figure 1 and supplementary information). Because of the energetic 
barriers between TPD and $\mathrm{Alq}_{3}$, holes tend to accumulate at the interface between the two materials. It should be noted that without any fixed negative charges at the interface between the $\mathrm{Alq}_{3}$ and the HTL, no holes would be present in the device for a negative applied voltage. In this regime, the OLED behaves like a Metal-Insulator-Semiconductor (MIS) device in the accumulation regime as described in [14], therefore the capacitance that is measured and simulated is close to the remaining capacitance of the $\mathrm{Alq}_{3}$ layer $\left(\mathrm{C}_{\mathrm{Alq} 3}\right)$.

For $\mathrm{V}=3 \mathrm{~V}\left(\mathrm{~V}>\mathrm{V}_{\mathrm{bi}}\right)$, both electrons and holes are injected and recombine at the internal interface, the OLED emits light.

The band diagrams described in figure 1 could in principle be validated using Electric-field-induced doubly resonant sumfrequency generation [15], in this technique, the electric field in the different layers of the OLED (and therefore the band diagram) can be measured by optical techniques.

Finally, an analytical expression relating the sheet charge density to the transition voltage can be found in [2]. The derivation of this analytical expression is done by finding the applied voltage corresponding to the transition voltage, at which the HTL is in flat band condition. This flat band condition in the HTL is not found at the built in potential ( $\mathrm{V}_{\mathrm{bi}}$ ), as usual, but at a lower voltage (the transition voltage) because the sheet charge density between the HTL and the ETL modifies the band diagram. Moreover, the same dielectric constant is assumed for both ETL and HTL in order to get equation (10), and $\mathrm{V}_{\text {tr }}$ can then be calculated as follows:

$V_{t r}=\frac{Q_{s}}{\epsilon} t_{E T L}+V_{b i}$

where $t_{E T L}$ represents the thickness of the ETL and $Q_{s}$ is the sheet charge density at the interface between the two materials. In this case $Q_{S}$ is negative, leading to a transition voltage which is below the built in potential.

\subsection{Capacitance-frequency measurements}

In a second step, we have measured the frequency response of the capacitance of a similar device: (ITO/PEDOT:PSS(30nm)/ $N, N^{\prime}$-Di(1-naphthyl)- $N, N^{\prime}$-diphenyl-(1,1'-biphenyl)-4,4'-diamine (NPB) (70nm)/Alq 3 (70nm)/Ca(15nm)/Al) for a fixed voltage $\mathrm{V}=0 \mathrm{~V}$ between $\mathrm{V}_{\mathrm{tr}}$ and $\mathrm{V}_{\mathrm{bi}}$ using PAIOS 2.2 [16]. The samples were processed as follows: first, the substrates were cleaned in an ultrasonic bath consecutively in technical isopropyl (10 min), clean acetone (10 min) and clean isopropyl (10 min). Before spin-coating PEDOT:PSS the samples were treated with UV-Ozone for 15 min to increase adhesion. PEDOT:PSS was spin-coated for $30 \mathrm{sec}$ and dried afterwards on a hot plate $\left(125^{\circ} \mathrm{C}\right)$ for 30 min. The thermal evaporation of the organic materials took place in an evaporation chamber under low pressure of $1 \times 10^{-7}$ mbar. The average evaporation rate 
for the organic materials was about $1 \AA$ As. For the measurement, the temperature was varied between $204 \mathrm{~K}$ and $299 \mathrm{~K}$ using a nitrogen cryostat connected to and controlled by Paios. Again two plateaus of the capacitance can be observed (figure 4). The high value of the capacitance corresponds to the geometrical capacitance of the $\mathrm{Alq}_{3}$ layer and the lower plateau corresponds to the geometrical capacitance of the full device. The transition frequency $\left(\mathrm{f}_{\mathrm{T}}\right)$, at which the capacitance changes from the first plateau to the second, strongly depends on temperature. This trend is nicely reproduced in simulations, when a hole injection barrier and/or a temperature dependent hole mobility is assumed. This suggests that the temperature dependence is related to the hole injection and transport, which is a temperature activated process. For the simplicity of the model we assume constant mobilities, so the observed temperature activation is due to the hole injection barrier. However, we can still notice slight differences between the simulated and measured C-f curves. First we observe, in the experiments, a rise of the capacitance at low frequencies, which does not appear in the simulations. This effect can be explained by the presence of a parasitic capacitance due to the un-patterned PEDOT:PSS layer as described in [3]. We also notice that the value of the higher plateau in simulations is slightly lower than in measurements, this can come from small variations of the layer thickness compared to their nominal values, moreover in simulations we have assumed a dielectric constant equal to 3.5 for both the NPB and the $\mathrm{Alq}_{3}$ layer which might slightly differ from real values.
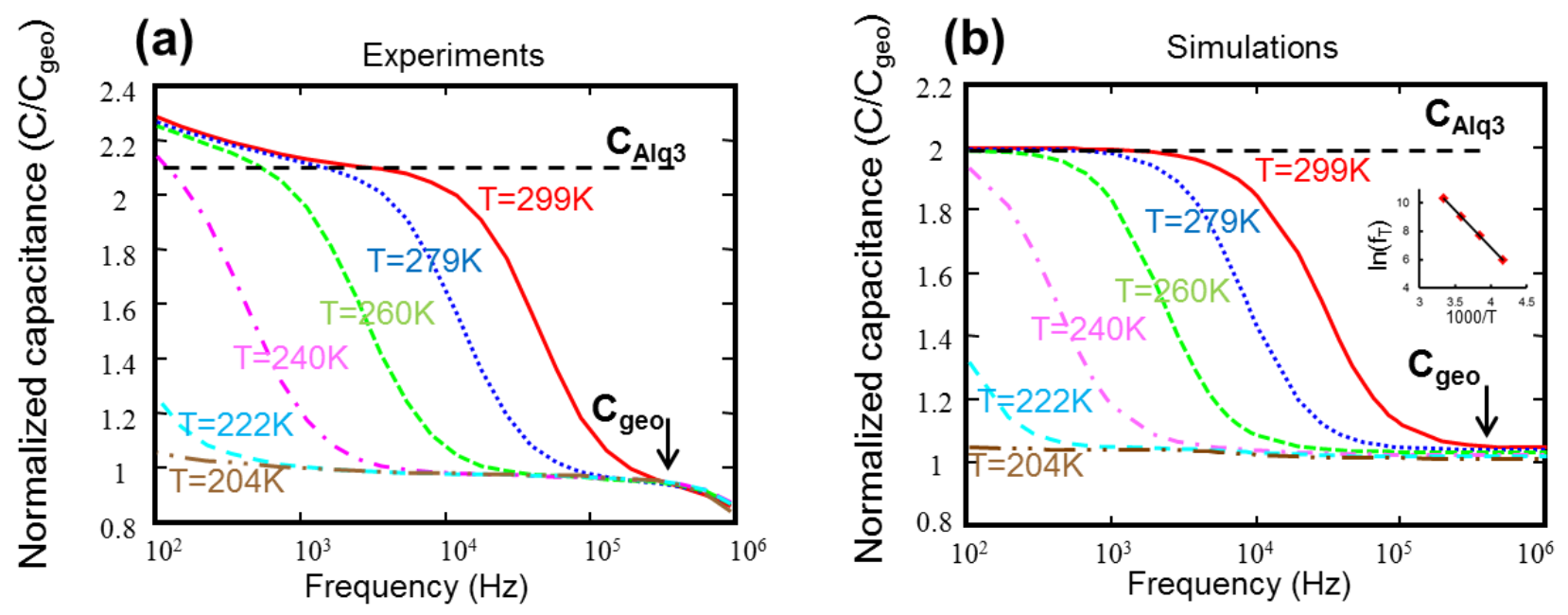

Fig.4 Measured (a) and simulated (b) capacitance versus frequency at different temperatures, showing the temperature activated hole injection. Inset, Arrhenius plot of the transition frequency versus the inverse of the temperature.

An activation energy of $0.45 \mathrm{eV}$ was extracted from the Arrhenius plot of the transition frequency versus the temperature. This value is very close to the injection barrier that is found in the literature between PEDOT:PSS and NPB and that was used for the simulations $(0.5 \mathrm{eV})$ : the workfunction of PEDOT:PSS is usually found between 4.8 and $5.1 \mathrm{eV}$ and the HOMO 
level of NPB usually lies around $5.4 \mathrm{eV}$ [2]. Simulations (not shown here) revealed that the hole concentration in the HTL layer strongly depends on the temperature due to the energetic barrier that the carriers have to overcome when 'hopping' from the anode into the NPB layer, the conductivity of this layer is thus strongly temperature dependent. However, from these measurements only, we cannot make conclusions concerning the activation energy of the mobility which might impact the overall activation energy; this point is out of the scope of this paper.

The formalism described in the modelling section of this paper (section 2) is also appropriate to simulate the capacitance of bilayer OLEDs where the ETL has an 'inverted polarity': with positive charges located at the interface with the HTL and negative charges located at the interface with the cathode. A bilayer device: ITO/ NPB(90nm)/ ETL(45nm)/ Ca(30nm)/ $\mathrm{Al}(70 \mathrm{~nm})$ was fabricated by conventional thermal evaporation at a base pressure of approximately $2 \times 10^{-6} \mathrm{mbar}$. All organic layers and metals were successively (in situ) deposited on a pre-cleaned ITO substrate. During the evaporation process, the vacuum chamber was kept in the dark to avoid possible influences on the spontaneous polarization orientation of the organic layers.

In this device, tris(7-propyl-8-hydroxyquinolinato) aluminum $\left(\mathrm{Al}(7-\mathrm{pyq})_{3}\right)$ serves as a polar ETL: indeed it has been demonstrated in [4] that the $\mathrm{Al}(7-\mathrm{pyq})_{3}$ material exhibits an 'inverted' polarity when deposited in an OLED.

The capacitance versus frequency and temperature was measured (figure 5) for an applied voltage of $-2 \mathrm{~V}$, as shown in figure 5. Again two plateaus are observed, and the transition frequency, at which the capacitance changes, strongly depends on the temperature, as observed previously. Again, all these trends can be nicely reproduced by the simulation (figure $5 \mathrm{~b}$ )).
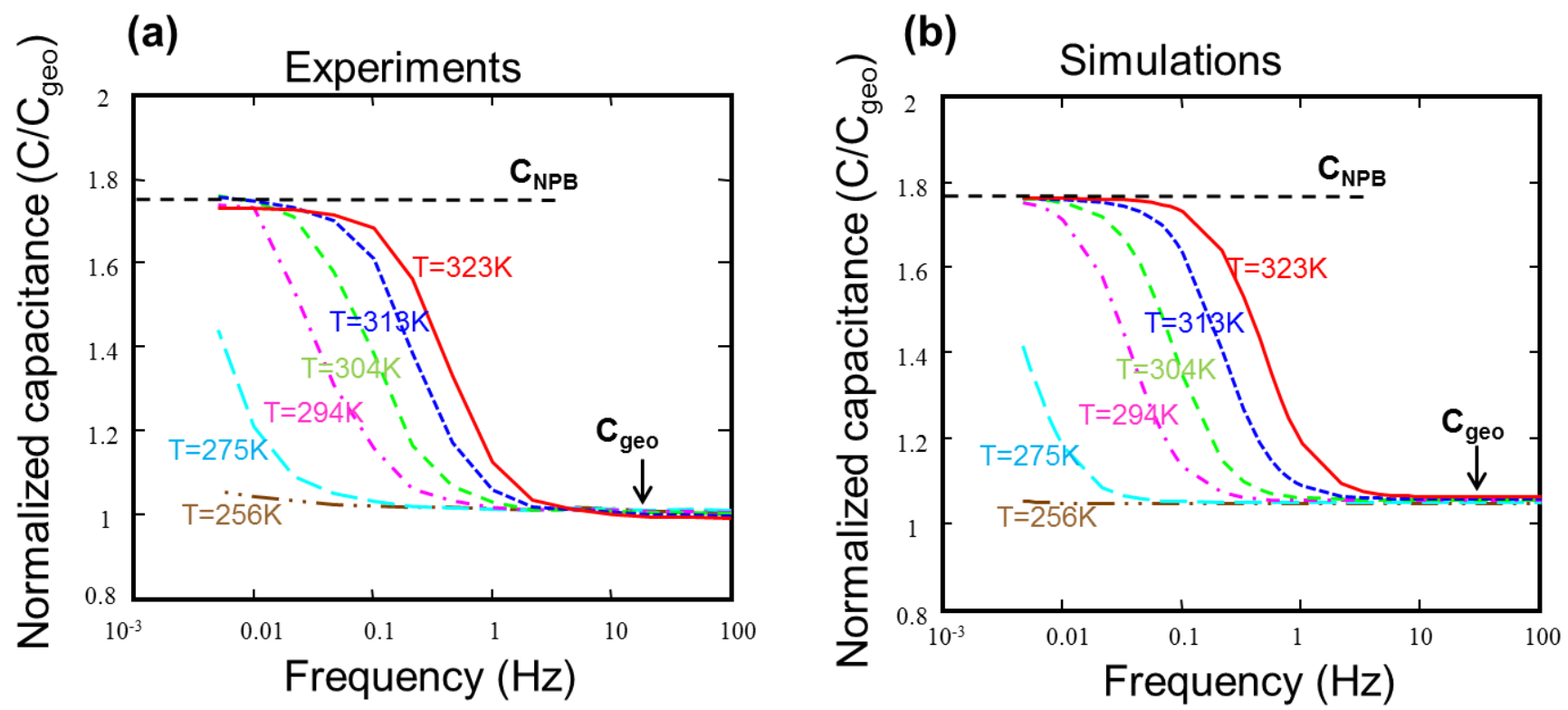
Fig. 5: Measured (a) and simulated (b) capacitance versus frequency of the OLED device with an inverted polarity at different temperatures.

The difference compared to the previous case is that, this time, the high capacitance plateau corresponds to the geometric capacitance of the HTL layer. Indeed, as shown in figure 6 , for an applied voltage of $-2 \mathrm{~V}$ holes are not injected in the device and remain in the vicinity of the anode. But, due to the positive charges at the HTL/ETL interface, electrons are injected from the cathode and accumulate at the HTL/ETL interface (Figure 6) even for a negative applied voltage (-2V). Therefore, for this applied voltage, we find again a MIS configuration, but this time the HTL serves as the insulator (instead of the ETL as found previously).

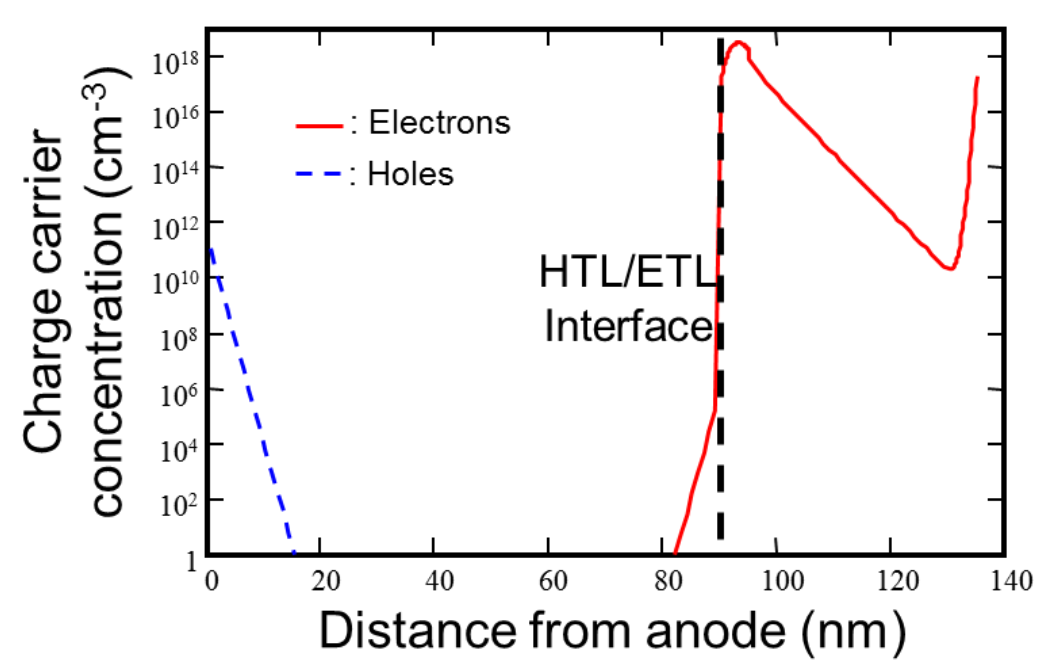

Fig. 6: Simulated electron and hole concentration in the OLED device with an inverted polarity for an applied voltage of - 2 V.

\section{Impact of the polarity of the ETL on the IV curve of the OLED}

In section 3, the accuracy of the model developed for simulating OLEDs with polar ETLs has been demonstrated by successful comparison between simulations and experiments. In figure 7 we show the simulated IV curve of a bilayer (ITO/PEDOT(30nm)/ NPB (70nm)/Alq $\left.{ }_{3}(40 \mathrm{~nm}) / \mathrm{Ca}(15 \mathrm{~nm}) / \mathrm{Al}\right)$ OLED device for three different cases: assuming a non-polar ETL, with a polar $\mathrm{Alq}_{3}$ and finally assuming a reversed polarity of the ETL (i. e. with a positive sheet charge density at the HTL/ETL interface). For the simulations, we have assumed a hole injection barrier of $0.5 \mathrm{eV}$ from the PEDOT:PSS to the $\mathrm{NPB}$ and an electron injection barrier of $0.3 \mathrm{eV}$ from the Calcium to the $\mathrm{Alq}_{3}$, since the electron affinity of the $\mathrm{Alq}_{3}$ is often found between $2.7 \mathrm{eV}$ and $3.0 \mathrm{eV}$ [4]. We can notice the increase of the current when the dipole moment of the ETL is 
directed toward the NPB layer and a decrease of the current when it is inverted. This latter point has been experimentally observed by Noguchi et al.[4], where the orientation of the molecular dipoles of the ETL could be inverted using the alternative electron transport material Al(7-pyq) 3 ). The sheet charge densities found in [4] (1.1 $\mathrm{mC}^{-\mathrm{m}^{-2}}$ for the Alq $\mathrm{q}_{3}$ and 3.1 mC. $\mathrm{m}^{-2}$ for the 'inverted' ETL) were used for the simulations shown in figure 7. In this simulation, the barrier of injection into the ETL was relatively small $(0.3 \mathrm{eV})$, for higher injection barriers we expect a bigger impact of the sheet charge density on the IV curve. From these simulations we can conclude that introducing a polar ETL with the proper orientation can be beneficial for the OLED as the current can be increased leading to a decrease of the driving voltage and therefore an increase in power efficiency.

(a)

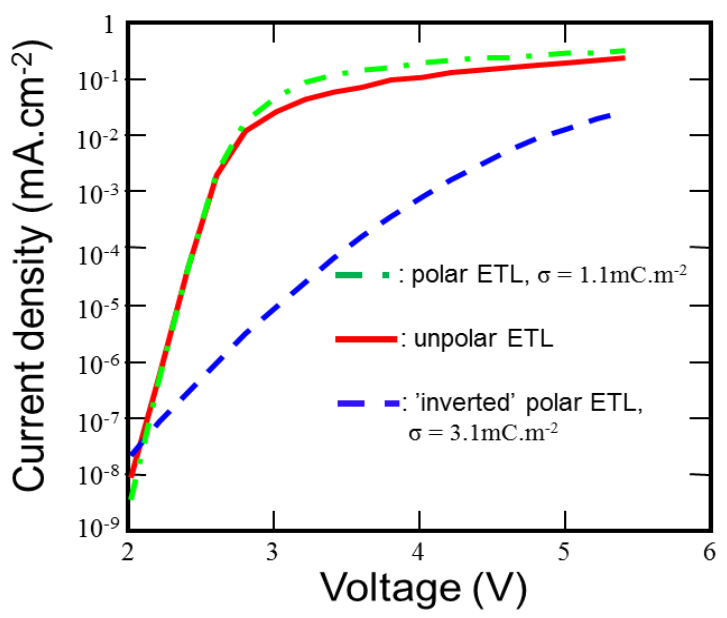

(b)

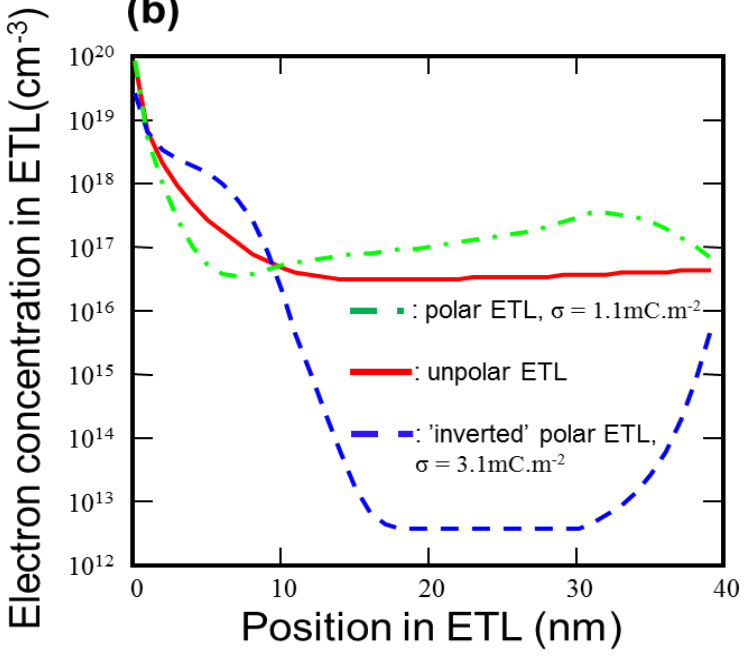

Fig.7: Simulated impact of the ETL polarity orientation on the IV curve of the OLED (a), and corresponding electron concentration in the ETL for an applied voltage of $3 \mathrm{~V}(\mathrm{~b})$.

In order to understand the reason why the current is modified by using a polar ETL material, we have simulated the electron concentration inside the ETL layer (figure $7 \mathrm{~b}$ )). We observe that when a negative sheet charge density is located at the HTL/ETL interface (and consequently a positive sheet charge density at the cathode/ETL interface), the concentration of electrons is increased at the cathode interface, leading to an enhanced injection inside the ETL and thus increasing the current. When the polarity is inverted, the electron concentration is decreased leading to a reduced conductivity of the ETL reducing the current flowing through the device. These findings are in agreement with the work from Kinjo et al.[17] where they studied the band diagram of $\mathrm{Alq}_{3}$ and $\mathrm{Al}(7-\mathrm{pyq})$ using photoelectron spectroscopy and photoelectron yield spectroscopy. 
Moreover, simulations have revealed that the charge balance in this device is, in all cases, equal to unity, meaning that all charges injected in the device will recombine with an opposite charge at the internal interface. Therefore from this observation, we can conclude that the polarity of the material has no impact on the recombination and the current efficiency of the device.

\section{Conclusion}

In this contribution we have shown that polar materials can be simulated using the commercially available simulation software SETFOS 4.3 by introducing charge sheets on both sides of the polar layer. The comparison of measured and simulated capacitance as a function of bias, frequency and temperature allows quantifying the polarity of the ETL material. Finally we have shown that the polarity of the ETL can be beneficial, if correctly oriented in the device as it can enhance the electron injection into the ETL layer.

This work was supported by the Swiss National Science Foundation (SNF) and the German Foundation for Reseach (DFG) [project number 151563].

\section{References:}

${ }^{1}$ S. Altazin, C. Reynaud, U. M. Mayer, T. Lanz, K. Lapagna, R. Knaack, L. Penninck, C. Kirsch, K. P. Pernstich, S. Harkema, D. Hermes and B. Ruhstaller (2015, June). SID Symposium Digest of Technical Papers Vol. 46, No. 1, San José U. S. A. (2015).

${ }^{2}$ W. Brütting, S. Berleb, A. G. Mückl, Org. El., 2 (1), 1-36 (2001).

${ }^{3}$ T. D. Schmidt, L. Jäger, Y. Noguchi, H. Ishii and W. Brütting. J. Appl. Phys., 117, 215502. (2015)

${ }^{4}$ Y. Noguchi, H. Lim, T. Isoshima, E. Ito, M. Hara, W. W. Chin, J. W. Han, H. Kinjo, Y. Ozawa, Y. Nakayma and H. Ishii, Appl. Phys. Lett., 102 (20), 203306 (2013).

${ }^{5}$ S. Nowy, W. Ren, A. Elschner, W. Lövenich, and W. Brütting, J. Appl. Phys., 107, 054501 (2010)

${ }^{6}$ Simulation software SETFOS version 4.3 by Fluxim AG, www.fluxim.com.

${ }^{7}$ G. F. Dibb, M. A. Muth, T. Kirchartz, S. Engmann, H. Hoppe, G. Gobsch, M. Thelakkat, N. Blouin, S. Tierney, M. Carrasco-Orozco, J. R. Durrant and J. Nelson. Scientific Reports, 3, 3335. (2013)

${ }^{8}$ F. Bloom, O. Langguth, T. Canzler, IDW Tech. Digest., 876-877, Sapporo Japan (2013)

${ }^{9}$ N. D. Nguyen, M. Schmeits and H. P. Loebl. Phys. Rev. B, 75, 075307-1 (2007).

${ }^{10}$ Setfos 4.3 Manual, p. 106 (2016)

${ }^{11}$ E. Knapp, B. Ruhstaller., J. Appl. Phys., 117 (13) 135501 (2015)

${ }^{12}$ E. Knapp, B. Ruhstaller., Optical and Quantum Electronics, 42 (11-13), 667-677 (2011)

${ }^{13}$ E. Ito, Y. Washizu, N. Hayashi, H. Ishii, N. Matsuie, K. Tsuboi, Y. Ouchi, Y. Harima, K. Yamashita and K. Seki., J. Appl. Phys., 92, 7306-7310 (2002)

${ }^{14}$ S. Altazin, R. Clerc, R. Gwoziecki, D. Boudinet, G. Ghibaudo, G. Pananakakis, I chartier and R. coppard., Org. El., 12, 897 ( 2011).

${ }^{15}$ T. Miyamae, N. Takada, and T. Tsutsui, Appl. Phys. Lett., 101, 073304 ( 2012).

${ }^{16}$ OLED and OPV characterization platform PAIOS version 2.0 by Fluxim AG, www.fluxim.com.

${ }^{17}$ H. Kinjo, H. Lim, T. Sato, Y. Noguchi, Y. Nakayama and H. Ishii, App. Phys. Express, 9, 021601 (2016). 

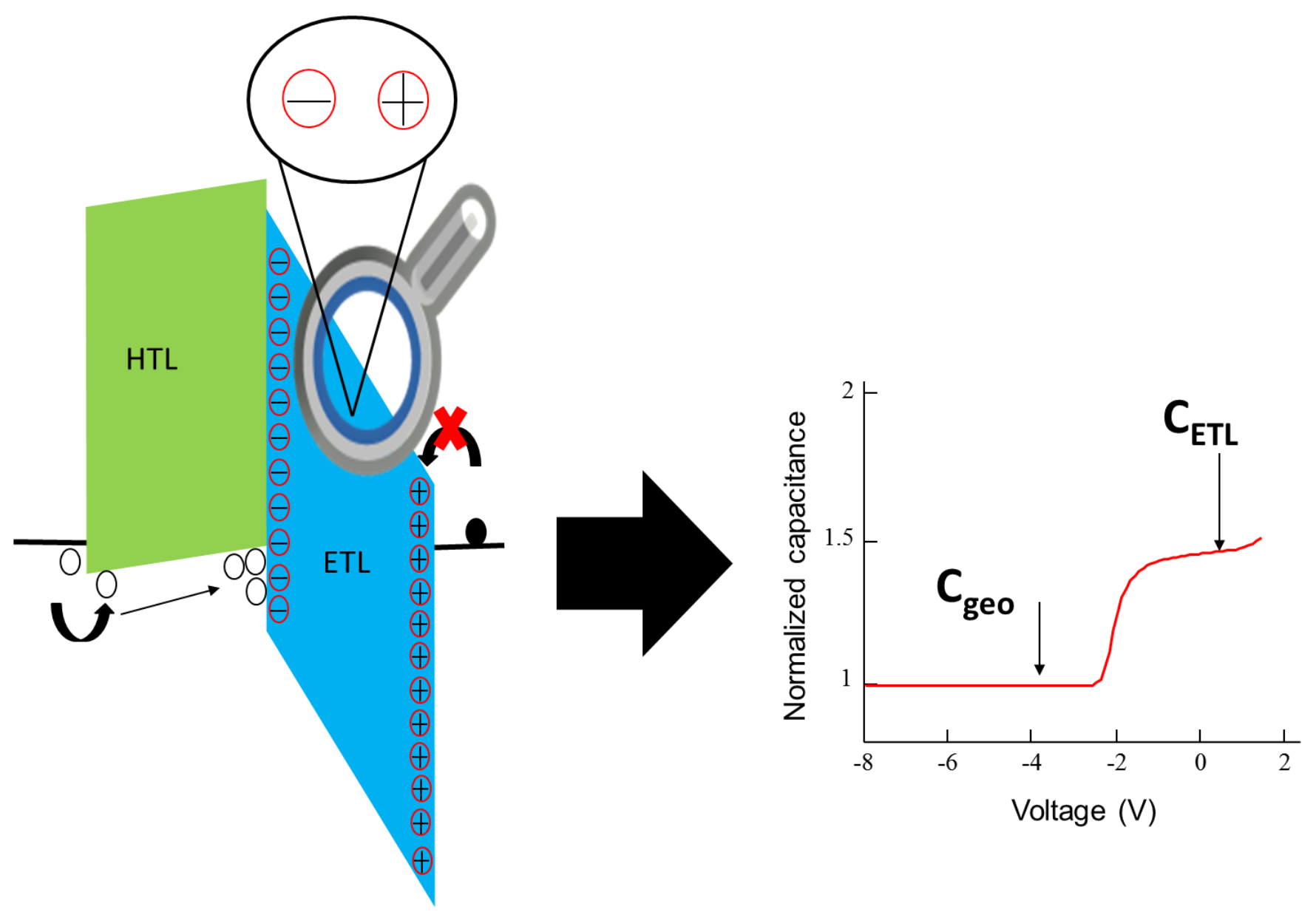